\title{
Einige Bemerkungen über die Beziehungen der Nerven und Muskeln zu den Centralorganen beim Embryo.
}

\author{
Von \\ Professor E. Neumanu \\ in Königsberg i. Pr. \\ Eingegangen am 29. August 1901.
}

Während sich der wichtige Einfluss, welchen die Centralorgane des Nervensystems auf die normale Ernährung der peripherischen Nerven and der Maskeln im extrauterinen Leben ausuiben, in unzweifelhafter Weise durch die Degenerationen und Atrophieen zu erkennen giebt, welche in letzteren eintreten, wenn ihre Verbindung mit Gehirn und Rückenmark unterbrochen wird oder diese selbst pathologische Veränderungen erlitten haben, muss es als eine noch offene Frage bezeichnet werden, in wie weit bei der embryonalen Entwickelung eine derartige »trophische Abhängigkeit der Nerven und Muskeln von den Nervencentren besteht, eventuell in welcher Entwickelungsperiode sie sich geltend zu machen beginnt; weder die pathologischen Erfahrungen über die Folgen von Entwickelungsstörungen der Nervencentren, noch die in neuerer Zeit vorgenommenen experimentellen Prüfungen haben ein befriedigendes Resultat ergeben. Ich habe diese interessante Frage bereits vor längerer Zeit in einem in dem Königsberger Biologischen Verein gehaltenen, jedoch nicht veröffentlichten Vortrage (19. Januar 1885), in welchem ich über meine Untersuchungsergebnisse bei der als Amyelie bezeichneten Missbildung berichtete, besprochen und sehe mich nunmehr zu einigen Bemerkungen über den gegenwärtigen Stand unserer Kenntnisse darüber veranlasst, da ich finde, dass in neveren Abhandlungen gewisse, durch gute ältere Beobachtungen gesicherte Thatsachen keine Berïcksichtigung gefunden haben und Anschaungen, welche diesen Thatsachen theilweise widersprechen, vertreten worden sind. 
Eine doppelte Beschränkung der Fragestellung seheint mir hierbei geboten. Erstens will ich von dem Verhalten der sensibeln Nerven absehen und nur die motorischen Bahnen sowie die Muskeln beriucksichtigen, weil für jene, die mit den Centralorganen gewissermaßen nur indirekt vermittels der in sie eingeschalteten Ganglien im Zusammenhange stehen, die Beurtheilung ihrer Beeinflussung durch Entwickelungsfehler der Nervencentra in Folge der dadurch gegebenen Komplikation viel größeren Schwierigkeiten unterliegt und überhaupt nur in solchen Fällen möglich erscheint, in denen sich eine gleichzeitige Betheiligung der Ganglien an der Entwickelungsstörung mit Sicherheit ausschließen lässt. Zweitens dürfte es sich aber anch empfehlen, mit Beiseitelassung des Gehirns vorzngsweise nur die Bildungsfehler des Rückenmarks ins Auge zu fassen, da die einstweilen vorliegenden Beobachtungen nur für dieses Organ ein geeignetes, auch mikroskopisch genau untersuchtes Material beigebracht haben und sich überdies voraussetzen lässt, dass die für die Rückenmarksnerven und ihre Muskeln geltenden Gesetze ohne Weiteres auch auf die aus dem Gehirnstamm entspringenden motorischen Nerven und die von ihnen versorgte Muskulatur übertragbar sind.

Unter den Bildungsfehlern des Rückenmarks unterscheiden wir aber füglich zwei Kategorien; die eine umfasst die Fälle, in denen die Störung so frühzeitig einsetzt, dass die Entstehung einer Rückenmarksanlage oder wenigstens einzelner Theile derselben ganz ausbleibt, in anderen, jedenfalls viel häufigeren Fällen dagegen wird erst später das in der Entwickelung begriffene Rückenmark in seiner weiteren Ausbildung gehemmt oder erfährt durch pathologische Processe mehr oder weniger tief eingreifende Veränderung. Dass die Rückwirkung auf motorische Nerven und Muskeln sich in dem einen und dem anderen Falle verschieden gestaltet, lässt sich von vorn herein vermuthen and sie verlangen daher eine gesonderte Prïfung.

Die einzigen, mir aus der Litteratur bekannten Beobachtungen, in denen sich mit fast unbedingter Sicherheit annehmen lässt, dass es sich um ein vitium primae formationis gehandelt habe, in Folge dessen die Bildung gewisser Abschnitte des Rückenmarks gar nicht zu Stande gekommen war, sind von keinem Geringeren als ERNST Heinrich Weber vor mehr als 50 Jahren in einem Aufsatze, welcher zuerst (1849) in den Abbandlungen der Leipziger Societät der Wissenschaften, sodann (1851) anch in MüLLER's Archiv veröffentlicht worden ist, mitgetheilt. Dieser Aufsatz, betitelt „Über die Abhängigkeit der Entstehung der animalischen Muskeln ron der der animalischen Nerven, 
erläutert durch eine ron E. H. Weber und Eduard WEBer untersuchte Missbildung", enthält außer der genanen Beschreibung einer eigenen Beobachtung gleichzeitig ein ausführliches Referat über zwei ähnliche, von ALEssandRIN in Bologna schon längere Zeit vorher beobachtete Fälle ${ }^{1}$ ), welche, ebenso wie der WEBER'sche, neugeborene Thiere betreffen. Der hohe Werth dieser bisher einzig dastehenden Beobachtungen ist namentlich darin begründet, dass die Übereinstimmung der Befunde, welche hier zwei zuverlässige Forseher unabhängig von einander erhoben haben, eine so große ist, dass man aus ihnen ohne Bedenken ein Gesetz ableiten kann; sie verdienen jedenfalls mehr Beachtung, als ihnen neverdings zu Theil geworden und es wird daher eine eingeheude Mittheilung über sie am Platze sein.

In WeBER's Falle lag ein reifes neugeborenes Kalb vor, dem der größte Theil der Wirbelsäule und damit zugleich der entsprechende Abschnitt des Rückenmarks nebst den zugehörigen Nerven und Muskeln gänzlich fehlte; Schädel und Halswirbel mit dem Gehirn und Halsmarke waren ausgebildet, von den Brustwirbeln war nur der erste normal, der zweite und dritte verkümmert, die übrigen fehlten gänzlich, ebenso sämmtliche Lendenwirbel, das Kreuzbein und die Schwanzwirbel. Das Riickenmark hörte am ersten Brustwirbel mit einem kugelig abgerundeten Knopfe, von dem die ersten Riickennerven entsprangen, auf, in den zweiten Brustwirbel erstreckte sich noch ein hohler Fortsatz der Dura mater, der dritte Brustwirbel war ohne Ruickenmarkskanal. Kopf und Hals, an denen die Gehirnnerven und die acht Halsnerven in ganz normaler Weise vorhanden waren, erschienen in allen Theilen wohl ausgebildet; auch an den vorderen Extremitäten bestand keine auffällige Veränderung bis auf eine unvollkommene Bildung der vou den Rippen entspringenden Muskeln, namentlich des Latissimus dorsi und des Serratus anticus major. Die Brust zeigte die sieben obersten Rippenpaare vorn mit dem Sternum verbunden, hinten aber, so weit die Wirbel fehlten, durch eine sehnige Haut mit einander zusammenhängend, achte und neunte Rippe standen weder mit der Wirbelsäule noch mit dem Brustbein in Verbindung, die untersten Rippen fehlten. Sämmtliche Brusteingeweide, ebenso das Zwerchfell sind gut gebildet, das Herz hatte zahlreiche Nerven, Nervi phrenici und vagi vorhanden, auch die Stämme des Sympathicus ließen sich

1) ALESSANDrins, An quinam nervi conferant ad evolutionem et incrementum systematis muscularis. Annali di Storia naturale 1829 und Novi commentari academiae scientiarum instituti Bononiensis. Tome III. 1839 (citirt näch Weber). 
Über d. Beziehungen d. Nerven n. Muskeln zu d. Centralorganen beim Embryo. 451

bis in den Anfang der Brusthöhle verfolgen. Der Banch (dessen Eingeweide leider, da sie bereits vorher entfernt waren, nicht zur Untersuchung gelangten) war ein Sack, der unten an den Beckenknochen angewachsen war und dessen Wände aus dem Peritoneum, einer fibrösen Haut und dem behaarten Fell bestanden, eine Muskelschichtaber nicht enthielten, wie auch die Rüekenmuskulatur großentheils fehlte. Das Becken eng, äußere und innere Geschlechtstheile fehlten, an dem (znrickgebliebenen) Endstiick des Mastdarmes zeigen sich eine Schleimhaut und »röthliche organische Muskelfasern «. Die Hinterbeine unbeweglich mit dem Becken verbunden, auch zwischen den einzelnen Knochen derselben fehlen die Gelenke, übrigens sind sie fast von 'natürlicher Länge, aber viel leichter und dünner als sie hätten sein sollen; ihr Pelz und die Hufe gut entwickelt, subcutanes Fettgewebe, Arterien, Venen, Lymphgefäße und Lymphdrïsen in normaler Weise vorhanden, aber Nerven und animalische Muskelfasern fehlen. „Die Sehnen einiger Muskeln wurden präparirt, z. B. die Achillessehne and die Sebnen der Streckmuskeln der Zehen, sie gingen ron den Knochen aus, denen die Muskeln angehören sollten, auf der anderen Seite endigten sie sich aber in sehnige Häute, an Stelle der Muskellamellen, welche sich an die sehnigen Häute ansetzen sollten, waren Fettlamellen vorhanden.\& Bei mikroskopischer Untersuchung zeigten sich in der Haut normale Haarbälge, Haardriisen und Schweißdriisen, Nervenfäden aber entdeckte man in ihr nirgends.

Auch der eine Fall von Alessandrini bezieht sich auf ein neugeborenes Kalb. Hier endigte die Wirbelsäule erst am zehnten Rückenwirbel und ging daselbst in ein einfaches, nicht sehr starkes Band über, welches die Verbindung mit dem Becken herstellte, es fehlten also mehrere Rückenwirbel, alle Lendenwirbel, das Kreuzbein und der Schwanz, der vorhandene Theil des Rückenmarks war natürlich gebildet, von ihm gingen 18 Nervenpaare, nämlich acht Halsnerven- und zehn Rückennervenpaare aus, in allen Theilen, zu welchen keine Rückenmarksnerven gelangten, feblten anch die willkürlichen Muskeln ganz, an Bauch waren nur diejenigen Theile der Muskeln sichtbar und von rother Farbe, welche noch von den Dorsalnerven Fäden erhielten, während an den Stellen, wo die Nerven fehlten, die Muskeln sogleich verschwanden; an der ganzen hinteren Körperhälfte existirten zwar Hant, Panniculus adiposus, Aponeurosen, Knochen and Blutgefäße, aber keine Muskeln. Die 
Eingeweide der Brust und des Unterleibes boten nichts Bemerkenswerthes, den Uterus ausgenommen, dessen Hörner in viele Zellen abgetheilt und angeschwollen waren. Aorta und untere Hohlvene mit ihren Ästen ziemlich normal, die Grenzstränge des Sympathicus hörten da auf, wo es keine Spinalnerven mehr gab, dagegen waren die Nervi splanchnici und die aus vielen Ganglien bestehenden Plexus solaris und mesenteriens, sowie die von ihnen ausgehenden Aste ungewöhnlich stark entwickelt.

Die zweite ganz ähnliche Missbildung, welche Aressaxdrins bei einem neugeborenen reifen Schweine vorfand, bot in so fern noch besonders interessante Verhältnisse dar, als Wirbelsäule sowohl als Riickenmark in Folge eines großen Defektes in zwei von einander getrennte Theile zerfielen, der obere Theil der Wirbelsäule bestand aus den sieben Halswirbeln und den sechs ersten Rïckenwirbeln und enthielt ein gut gebildetes Rückenmark mit 13 Nervenpaaren, der untere aus den Schwanzwirbeln, von denen die vier obersten in ihrem Kanal eine kleine isolirte Abtheilung des Riickenmarks mit den Ursprïngen von vier Schwanznervenpaaren umschlossen. Demnach fehlte auch hier in der unteren nicht mit Rückenmarksnerven versorgten Körperhälfte die Muskulatur, aber der Schwanz war mit Muskeln versehen; am Bauche zeigten sich nur die oberen Theile einiger Muskeln, nämlich des Rectus und Obliquus externus, ausgebildet, die unteren fehlten; die Stelle der Muskeln nahmen ein übermäßig vermehrtes Zellgewebe und die Blutgefäße ein. Die Knochen der Hinterbeine waren kleiner als im natürlichen Zustande, übrigens aber wohlgebildet, die Gelenke sämmtlich steif $»$ wahrscheinlich wegen des Mangels der Muskelfasern, denn unter diesen Umständen waren die Gelenke während des Lebens des Fötus niemals in Bewegung versetzt worden «. Die Integumente hatten am vorderen Theile des Körpers, wo sie mit zahlreichen Nerven verseben waren, und am hinteren, wo diese ganz mangelten, dasselbe Aussehen und man konnte nicht einmal zwischen der die Hautwärzchen bildenden Schicht an beiden Gegenden eine Verschiedenheit wahrnehmen«. - Am Schädel bestand Cyklopenbildung, Bauch- und Brusteingeweide waren entfernt und konnten nicht untersucht werden.

Auch vom menschlichen Embryo existirt eine ähnliche, wenn auch nicht gleich prägnante Beobachtung, die von BaRKow ${ }^{1}$ )

1) Barrow, Beitr. zul pathol, Entwickelungsgeschichte. Breslan 1854. 
Über d.Beziehungen d. Nerven u. Muskeln zu d. Centralorganen beim Embryo. 453

herrührt, und einen hohen Grad unvollständiger Entwickelung des Muskelsystems und gänzlichen Mangel des Zwerchfells betrifft. Ich entnehme SaMugl's bekanntem Aufsatz über Trophoneurosen ${ }^{1}$ ) darüber Folgendes: Die großen Hirnhemisphären waren durch Hydrocephalus in zwei große Säcke umgewandelt, deren Wände durch Pia mater, Arachnoidea und einige Linien dicke Lage von Hirnsubstanz gebildet wurden; das kleine Gehirn, das verlängerte Mark von geringem Umfang, das Rückenmark stieg bis an den vierten Lendenwirbel herab, war aber von geringer Breite. Die vorderen und hinteren Wurzeln der Rückenmarksnerven waren zwar alle vorhanden, aber außerordentlich dünn, nur sehr feine Fäden darstellend, die Spinalganglien von Hirsekorngröße; während die N. optici, acustici, olfactorii vorhanden waren und die höheren Sinnesorgane auch wenig Anomalien darboten, fehlten von Hirnnerven Oculomotorins, Trochlearis, Glossopharyngeus und Accessorius gänzlich, die N. trigemini waren sehr unbedeutend, Eingeweide incl. Herz und Gefäßsystem boten nichts Besonderes. Die Nervi phrenici fehlten gänzlich an beiden Seiten, dementsprechend fehlte das Zwerchfell vollständig; die Rückenmarksnerven waren höchst unbedeutend oder fehlten gänzlich, dementsprechend fehlten alle willkürlichen Muskeln entweder ebenfalls oder bestanden nur aus Muskelsubstanz, welche mit sehnigen Fasern und viel Fett durchwachsen war.

Die Deutung; welche Wrake den von ihm berichteten Beobachtungen gegeben hat, dünkt mich unanfechtbar. Er geht davon aus, dass zufällige äußere Einwirkungen die regelmäßige Bildung des Rückenmarks und der Wirbelsäule zu einer Zeit gestört hatten, als die erste Anlage dieser Organe entstand und die Rückenmarksnerven noch nicht gebildet waren und nimmt ferner an:

1) dass die Bildung der Rückenmarksnerven in einer gewissen Abhängigkeit von den schon gebildeten Rückenmarkstheilen erfolgt, demnach also unterbleibt an den Orten, wo letztere nicht existiren,

2) dass ebenso eine Abhängigkeit der Bildung der Muskelfasern von der der Nerven besteht, so dass als Folge der verhinderteu Bildung der Nerven eine Nichtbildung der Muskelfasern sich zeigt.

Eine andere Annahme zur Erklärung des in Verbindung mit den Rïckenmarksdefekten beobachteten Fehlens der Muskeln und Nerven,

1) Samuel, Trophoneurosen, in der Realencyklopädie f. d. ges. Heills. Sep.-Abdr. pag. 7. 1885. 
nämlich die, dass die störende Ursache, welche auf das Rückenmark eingewirkt hatte, gleichzeitig auch den »Bildungsstoff «, aus welchem die Rückenmarksnerven mit ihren $\ddot{A} s t e n$ und die Muskeln ihren Ursprung nehmen, betroffen habe, hat WEBER wohl erwogen, aber mit vollem Recht entschieden zurückgewiesen, weil dann auch die Bildung der neben den Nerven liegenden Blutgefäße verhindert worden wäre. Später ist allerdings CoHNHem ${ }^{1}$ ) für eine derartige Auffassung eingetreten, man wird aber SAMUEL ${ }^{2}$ ) beistimmen müssen, wenn er dieselbe trotzdem für haltlos erklärt and hervorhebt, dass, „wenn die Entwickelung einer Extremität im Übrigen ungestört, die Muskulatur allein durch Fettgewebe ersetzt ist, keine Ursache gleichzeitig die Entwickelung des Rückenmarks resp. seiner Nerven und die der ganz entfernten Muskulatur völlig gehemmt, das ỉbrige Gewebe der Extremität dabei aber gänzlich intakt gelassen haben kann", ron einem "Koeffekt einer Störung in der Anlage beider" im Sinne ComsHEIM's also nicht die Rede sein könne. Wir dürfen von einem solchen hypothetischen Erklärungsversuche, wenigstens was den Mangel der Nerven betrifft, um so eher absehen, als ja die von Weber aus seiner Beobachtung erschlossene Abhängigkeit der Bildung derselben von den Centralorganen durch die neueren embryologischen Forschnngen zu einer unzweifelhaften Thatsache geworden ist, wir sind durch dieselben sogar in den Stand gesetzt, mit annähernder Sicherheit den Zeitpunkt anzugeben, in welchem der partielle Untergang. der Rückenmarksanlage stattfand; da nämlich mit dem Rückenmark nicht nur die vorderen, sondern zugleich auch die hinteren Wurzeln mit den Spinalganglien and sämmtliche peripherischen Nerven fehlten, so müssen wir wohl annehmen, dass die Störung eintrat nicht nur bevor die vorderen Wurzeln aus dem Medullarrohr hervorwuehsen, sondern auch ehe noch die Spinalganglien sich aus ihm ausgesondert hatten, anderenfalls wäre der Ausfall nur auf das System der motorischen peripheren Bahnen beschränkt geblieben.

Der erwähnte zweite WEBER'sche Satz, betreffend die Abhängigkeit der Entstehung der Muskeln von der vorhergehenden Bildung. der zugehörigen Nerven, ergiebt sich freilich nicht unmittelbar aus den bisher bekannten entwickelungsgeschichtlichen Daten $\left.{ }^{3}\right)$. Ein

1) Connheim, Allgemeine Pathologie. 2. Aufl. I. pag. 594.

2) Samuer, 1. c. Sep-Abdr. pag. 8.

3) Der von Nussbaum (Verhandl. d. Anat. Gesellsch. 1894. pag. 180. Arehiv f. mikr. Anat. Bd. 47. pag. 416. 1896) geführte Nachweis, dass bei Anurenlarven die Muskeln von der Nerveneintrittsstelle aus in der Richtung, welche die in 
Abhängigkeitsverhältnis der Muskeln von den motorischen Nerven in der Art, dass erstere aus letzteren hervorwachsen, wie die Nerven ans Gehirn und Rïckenmark, besteht sicher nicht; wir können, wenn ein Abhängigkeitsverhältnis wirklich besteht, uns die Beeinflussung der Muskelentstehung durch die Nerven nur als eine dynamische vorstellen in dem Sinne, dass das ursprünglich indifferenzirte Bildungsmaterial der Muskeln nur dadurch seine specifische Prägnng erhält, dass es mit den ans den Centren hervorwachsenden Nerven in innigen Kontakt geräth, dass also zwischen beiden Organen, um einen neuerdings beliebten Ausdruck zu gebrauchen, eine Entwickelungskorrelation besteht.

Ich stehe nicht an, da mir eine andere Erklärung der WEBERAlessandRINI'schen Beobachtungen ausgeschlossen zu sein scheint, in denselben einen vollgültigen Beweis für die Existenz einer solchen Korrelation zu erblicken, der um so werthvoller ist, da zur Zeit weder durch die Erforschung der normalen Entwickelungsvorgänge noch durch andere pathologische Befunde oder experimentelle Erfahrungen an Thierembryonen ein solcher Nachweis hat gefuihrt werden können. Neuere Autoren haben meiner Ansicht nach mit Unrecht auf Grund ihrer Experimente an Amphibienlarven, bei welchen sie Defekte der Nervencentra erzeugten, ganz im Allgemeinen die Annahme einer »korrelativen Entwickelung benachbarter Organe oder einer funktionellen Kontrolle der Gesammtentwickelung durch ein Centralorgan « verworfen. SCHAPER ${ }^{1}$ ) zieht aus seinen Versuchen den Schluss, dass »während einer frühen Entwickelungssperiode das Centralnervensystem keinerlei funktionellen Einfluss auf die vitalen Vorgänge im sich entwickelnden Organismus ausiibt und weder specifische centripetale Reize aufnimmt, noch irgend welche specifische Reize centrifugal aussendet, $d$. $h$. dass es weder sensible noch motorische noch morphogenetische Funktionen hat; . . . . alle Wachsthums- und Differenzirungsvorgänge vollzogen sich nach typischen Principien $\ll$. Dem schließt sich Barfur'rh ${ }^{2}$ ) an, indem er

ihrer Anlage sich verbreitenden Nerven nehmen, wachsen und dass somit die Enden der Nervenzweige die juingsten Partien der Muskeln bezeichnen, verdient in dieser Beziehung zwar die größte Beachtung, es geht aus ihm aber nicht nothwendig hervor, dass das Mnskelwachsthum die Folge der weiter vorschreitenden Nervenverbreitung ist, es könnte sich dabei ja um zwei einander parallel gehende, aber von einander unabhängige, a uton ome Vorgänge handeln.

1) A. Schaper, Experimentelle Stndien an Amphibienlarven. Erste Mittheilung. Archiv f. Entwickelungsmech. Bd. VI. pag. 182. 1898.

2) Barforth, Sitzungsber. d. naturforsch. Ges. in Rostock. 1901. Nr. 1. 
mit Bezug auf die Schaper'schen Experimente und auf Grund eigener, die Regenerationsprocesse bei defekten Froschlarven betreffenden Versuche ganz allgemein sagt; " für die normalen Entwickelungsund Wachsthumsvorgänge früher: Stadien kann ein regulirender und trophischer Einfluss des Nervensystems ausgeschlossen werden «. Beide Autoren treten demnach für das Roux'sche Princip der Selbstdifferenzirung aller Theile als ein für die frühere Embryonalperiode allgemein gültiges Gesetz ein.

Die Weber-Alessandrint'schen Fälle scheinen Schaper und Barfurth jedoch unbekannt geblieben zu sein, wenigstens finden sie keine Erwähnung, obwohl der Erstere in seiner ausführlichen Arbeit vielfache Litteraturnachweise giebt und auch mehrere pathologische Beobachtungen heranzieht. Nur dadurch scheint es mir erklärlich, dass beide der Annahme einer Entwickelungskorrelation zwischen Nervencentren und anderen Organen gegenüber einen unbedingt negirenden Standpunkt einnehmen, sie hätten sonst sicher erkannt, dass jene Beobachtungen ein von der Natur selbst in großem Maßstabe durchgeführtes Experiment darstellen, dessen klarer Erfolg in ein em Punkte im Widerspruche steht mit den Schlüssen, welche sie aus ihren Versuchen gezogen haben, indem sie nämlich zeigen, dass wenigstens ein Gewebe im menschlichen Körper existirt, dessen Entwickelung schon in frühester Zeit eines Impulses von Seiten eines Centralorgans bedarf, nämlich das Gewebe der quergestreiften Muskelfasern, und dass also hier das Princip der Selbstdifferenzirung seine Grenze findet; es wäre ihnen auch der Grund nicht entgangen, wesshalb ihre Experimente zu einem abweichenden Resultat führten, derselbe muss offenbar darin gesucht werden, dass der von ihnen ausgeübte Eingriff in einen späteren Zeitpunkt der Entwickelung fiel, als der pathologische Vorgang, welcher den in der WeBER'schen Mittheilung beschriebenen Missbildungen zu Grunde lag. Bei letzteren waren Theile des Medullarrohres zerstört worden zu einer Zeit, als ein peripherisches Nervensystem noch gar nicht bestand, SchaPER and BARFurTH dagegen operirten bei Larven, bei welchen Nerven und Muskeln bereits in ihrer Anlage vorhanden waren und nur einer weiteren Ausbildung harrten. Thre Versuche waren demnach wohl geeignet, Aufschluss über die Frage zu geben, welchen Einfluss ein Fehlen der nervösen Centralorgane auf den Bestand und das Wachsthum der bereits entstandenen peripheren Nerven und Muskeln während der embryonalen Entwickelung: ausübt und sie liefern, wie aus dem Folgenden sich ergeben wird, werthvolle Beiträge zur Lösung dieser Frage, sie können aber nicht 
zur Entscheidung darüber, ob die erste Entstehang peripherer Nerven und Muskeln unabhängig von den Nerwencentren erfolgt, herangezogen werden.

Die von WeBEr aus seinen und ALEsSANDrini's Beobachtungen gezogenen Schlussfolgerungen bleiben somit zu Recht bestehen, ebenso wie die Entstehung der motorischen Bahnen von den Nerrencentris auggeht and ihre Präexistenz voraussetzt, ebenso können die willkürlichen, quergestreiften Muskelfasern nur sich bilden, wenn vorher die motorischen Bahnen angelegt sind. Übrigens sei darauf hingewiesen, dass gerade diese WEBER'schen Fälle das für die anderen Gewebe gültige Princip der Selbstdifferenzirung in eklatantester Weise zum Ausdruck bringen und als wichtigste Stiutze desselben gewiss schon oft erwähnt worden wären, wenn sie allgemein bekannt gewesen wären und nicht bisher eine ziemlich verborgene Existenz geführt hätten. Was kann in der That besser die Selbständigkeit der Entwickelungsvorgänge in den einzelnen Theilen illustriren als die Thatsache, dass bei jenen Missbildungen auch da, wo Nerven gänzlich fehlten, nicht nur die gröbere anatomische Anordnung sämmtlicher Organe, Haut, Knochen, Fettgewebe, Blut- und Lymphgefäße, ja selbst der zu den fehlenden Muskeln gehörenden Sehnen eine vollständig normale war, sondern auch die feinere mikroskopische Struktur in ihnen, abgesehen von dem Mangel der Nerven, kaum einen Untersehied gegenüber den mit Nerven versehenen Körperabschnitten erkennen lieb?

Diesen ganz vereinzelt dastehenden pathologisehen Beobachtungen steht nun gegenuiber die große Gruppe derjenigen Bildungsfehler der Nervencentra, in welchen dieselben erst, nachdem sie eine gewisse Stufe der Ausbildung erreicht und das periphere Nervensystem sowie die Muskulatur gleichfalls zur Entwickelung gekommen, in ihrer normalen Weiterentwickelung gehemmt oder durch pathologische Processe wieder zerstört worden. Hierher gehören ebensowohl die als Anencephalie und Amyelie bezeichneten Missbildungen, als auch die meisten Fälle sogen. Spina bifida oder partieller Rhachischisis. Was lässt sich aus dem hierüber vorliegenden sehr umfangreichen Material über den Zustand der Nerven und Mnskeln entnehmen? Eine Durchsicht der Litteratur zeigt leider, dass die Zahl der einer genanen Untersuchung in Bezug auf diesen Punkt unterworfenen Fälle keine sehr große ist und dass in mehrfacher Beziehung Widerspriche zwischen den Angaben verschiedener Untersucher bestehen. Gerade aber hier liefern einige Experimente einen gewissen Ersatz. 
Am klarsten liegen die Verhältnisse bei den Muskeln. Es ist eine von Alters her bekannte Erfahrung, dass bei anencephalen (hemicephalen) and amyelischen Friichten auch in solchen Fällen, wo Gehirn und Rückenmark vollständig zu fehlen scheinen, die Entwickelung des ganzen Körpers einen hohen Grad erreichen kann, so dass sie zur Zeit der Geburt in Bezug auf Körperdimensionen und Gewicht normalen Nengeborenen, welche die volle Reife erlangt haben, keineswegs nachstehen. Schon hieraus lässt sich erschließen, dass auch die Muskulatur keine Einbuße erlitten hat, und bei der Untersuchung findet man sie in der That kräftig entwickelt, von normaler rother Farbe, mikroskopisch mit typisch ausgeprägter Struktur. Ebenso steht es fest, dass bei der Spina bifida mit dem partiellen Defekt des Rückenmarks ein Mangel oder eine Degeneration der korrespondirenden Muskeln nicht verbunden za sein pllegt. Schou E. H. WEBER hatte in seiner mehrfach erwähnten Abhandlung, im Anschluss an die vorhin besprochenen merkwürdigen Beobachtungen, zugleich die Beschreibung eines Embryo gegeben, »dessen Gehirn und Rückenmark gänzlich fehlten, während die Nerven bis an ihre Wurzeln vorhanden, die Mnskeln ausgebildet und der Embryo bis zur Geburt vollständig ernährt worden war «, hier wird auch bereits der Gegensatz hervorgehoben, welcher im Vergleich mit jenen anderen Missbildungen hervortritt. WEBER's Schlussfolgerung ans dieser Beobachtung geht dahin, dass, wenn die Rückenmarksnerven und die Muskeln bei dem Embryo erst entstanden sind, sie za ihrer Ernährung und ihrem Wachsthum den Einfluss des Rückenmarks nicht mehr nöthig haben, und selbst eine völlige Zerstörung desselben ihre Ernährung nicht beeinträchtigt. Es wird zu prïfen sein, ob sich diese Auffassung anch gegenwärtig aufrecht erhalten lïsst.

Die Sachlage hat sich seit der Zeit, als WEBER seine Mittbeilung machte, in so fern wesentlich verändert, als damals der trophische Einfluss, welchen das Rückenmark auf die aus ihm hervorgehenden motorischen Nerven und die von diesen versorgten Muskeln im späteren extrauterinen Leben ausübt, noch unbekannt war; noch 20 Jahre später war die Beziehung, welche die großen Vorderhornzellen des Rückenmarks zu Nerven und Muskeln haben, so wenig ein in Rechnung zu ziehender Faktor, dass MaNz') in seinem 1870 erschienenen Aufsatze "Das Auge der hirnlosen Missgeburten den

1) MANz, VIRChOW's Archiv. Bd. 51. 
auch von ihm beobachteten unversehrten Zustand der Nerven und Muskeln bei anencephalen und amyelischen Früchten auf ihre erhaltene Verbindung mit den Spinalganglien zurückzuführen suchte, nachdem inzwischen WaLler ${ }^{1}$ ) und KuettNer ${ }^{2}$ ) die trophische Bedentung der Ganglienzellen richtig erkannt und letztere als »neurogenotrophe Organe in demselben Sinne, welcher mit dem zur Zeit geläufigen Ausdrucke » trophische Centren « (CHarco'T) verknüpft ist, bezeichnet hatten. Wenn wir jetzt der Frage näher treten, wie es zu erklären ist, dass trotz bestehender sAmyelie « die Muskeln nicht nur erhalten bleiben, sondern sogar frei von allen Erscheinungen einer degenerativen Atrophie sich zeigen, so sind wir darauf angewiesen, uns zwischen zwei sehr verschiedenen Anurahmen zu entscheiden: entweder ist die trophische Einwirkung, welche im späteren extrauterinen Leben von den Ganglienzellen der Vorderhörner des Rückenmarks auf Nerven und Muskeln ansgeübt wird, in der Embryonalperiode noch nicht vorhanden, oder es sind in solchen Fällen trotz des Anscheins der Amyelie jene Ganglienzellen verschont geblicben und stehen durch normal entwickelte motorische Nervenfasern mit den Muskeln in Verbindung; die erstere Annahme würde mit der WEbER'schen Auffassung übereinstimmen und nur eine präcisere Formulirung derselben darstellen; um sie zurückweisen zu können, würde also der Nachweis gefïhrt werden müssen, 1) dass bei sogen. Amyelie immer Ganglienzellen in Rückenmarksresten erhalten bleiben, und 2) dass auch stets leitungsfähige motorische Bahnen die Verbindung mit den Muskeln herstellen.

So weit ich das vorliegende Material uibersehe, sind diese beiden Anforderungen nicht erfüllt. Ganz abgesehen davon, dass Fälle von Amyelie beschrieben worden sind, in welchen die vorderen Rückenmarkswurzeln und mit ihnen die gesammten motorischen Bahnen der peripherischen Nerven gefehlt haben sollen - eine Angabe, auf welche nochmals zurickgekommen werden soll - scheint es auch nicht zweifelhaft, dass mit der Anomalie hänfig auch ein mehr oder weniger vollständiger Verlust der Ganglienzellen des Rüickenmarks verbunden ist. Dass es zur Feststellung dieses Punktes einer genanen mikroskopischen Untersuchung der Rückenmarksreste bedarf, ist selbstverständlich, und es sind daher alle älteren Fälle, wo eine

1) Warcer, Nouvelle méthode anatomique pour l'investigation. MÜLLER's Archiv. 1852.

2) Kuetrines, De origine n. sympathici ranarum ex nervi dissectione dijudicanda. Diss. Dorpat 18504. 
solche Untersuchung unterblieben ist, für die Beurtheilung dieser Frage unbrauchbar. Fine entscheidende Bedeutung dürfte folgenden Beobachtungen zukommen.

Leonowa ${ }^{1)}$ beschrieb einen Fall von Anencephalie kombinirt mit totaler Amyelie, in welchem Gehirn und Rïckenmark vollständig fehlten, und hebt ausdriicklich als Ergebnis der mikroskopischen Untersuchnng hervor, dass, »obwohl die Vorderhornzellen und die vorderen Rückenmarkswurzeln total fehlten, sich die quergestreiften Muskelfasern frei ron irgend welchen Veränderungen zeigten ".

FraSER ${ }^{2}$ ) fand bei der anatomischen Untersuchung eines ähnlichen Falles gleichfalls vollständigen Mangel der Nervencentra und konstatirte insbesondere wthe absence of the ventral cells in the central nervous system ", sowie sthe presence of a fully developed striated muscular system " und schließt daraus, dass "the roll of the ventral cells in the embryonic cord must be a very different on/to that generally attributed to it in the fully formed cord «.

Die Brïder C. und $G$. Petrén ${ }^{3}$ ) ferner überzeugten sich bei einem $29 \mathrm{~cm}$ langen Embryo mit Anencephalie und Amyelie, dass auch die mikroskopische Untersuchang »keine Spur von Rückenmark " erkennen ließ, und sagen, dass sie »die sehr bemerkenswerthe Beobachtung von Leovowa haben bestätigen können, dass die Muskelfasern trotz der vollständigen Abwesenheit der motorischen Ganglienzellen, der vorderen Wurzeln und aller Wahrscheinlichkeit nach auch des größten Theils der motorischen Nervenfasern eine normale Ausbildung erreicht haben", sie können in Übereinstimmung mit Leonowa diese Thatsache nicht anders deuten als so, »dass der Einfluss der motorischen Nerven auf die Ernährung der Muskeln nicht in der frühesten Entwickelungsperiode vorhanden ist, sondern sich erst später einstellt«.

Ein vierter ähnlicher Fall, von GaDE $\left.{ }^{4}\right)$ publicirt, ist mir im Original nicht zugänglich, nach den Angaben von C. und G. PetréN hat derselbe jedoch zu dem gleichen Ergebnis geführt.

1) Leonowa, Zur pathologischen Entwickelung des Centralnervensystems. Neurolog. Centralbl. 1893. pag. 220.

2) Fraser, An various single and double monstrosities with remarks on anencephalic and amyelic nervons systems. Trans. R. Acad. of Med. Irland. XII. 1895 (citirt nach SCHAPER, 1. c. pag. 186).

3) C. und G. Petrín, Beitrag zur Kenntnis des Nervensystems und der Netzhaut bei Anencephalie und Amyelie. Vinchow's Archiv. Bd. 151. Heft 2 und 3. 1898.

4) GaDE, Norsk Magazin for Laegevidenseaben. 55. Aargang 1894. 
Über d. Beziehungen d. Nerven u. Muskeln zu d. Centralorganen beim Embryo. 461

An diese pathologischen Beobachtungen schließen sich die von SCHAPER (l. c.) auf experimentellem Wege gewonnenen Resultate bestätigend an. SchAPER hatte bei einer $6 \mathrm{~mm}$ langen Froschlarve fast das ganze Gehirn operativ entfernt, nach $6 \frac{1}{2}$ Tagen, als das Thier getödtet wurde, war es um $2 \mathrm{~mm}$ (d. h. nur etwas weniger als das Längenwạchsthum normaler Vergleichslarven in diesem Zeitraum betrug) gewachsen, bei der Untersuchung zeigte sich, dass das Rückenmark eine so vollständige Atrophie erlitten hatte, dass die Larve am Ende der Beobachtung nicht nur als anencephal, sondern auch als "amyelitisch" betrachtet werden konnte und somit »von einem gewissen Zeitpunkt an das gesammte Centralnervensystem funktionell aus dem Körper ausgeschaltet war «. Trotzdem, »bei vollständiger Abwesenheit der motorischen Hirncentren und der Atrophie der Vorderhornzellen des Rückenmarks", konstatirte ScHaPer, dass die Muskulatur sowohl morphologisch zn vollkommener Entwickelung gelangt als auch histologisch mehr oder weniger zu derselben Höhe als in der normalen Larve differenzirt war, er fügt hinzu, ses muss dies um so mehr Wunder nehmen, als wir doch wissen, dass in postembryonaler Zeit Centralnervensystem und willkürliche Muskulatur in so enger Wechselbeziehung stehen, dass Vernichtung eines circumscripten Abschnitts des einen Organs unfehlbare Degeneration einer entsprechenden Partie des anderen Organs im Gefolge hat". Wenn ich dieser Beobachtung nicht ganz dieselbe Beweiskraft zuerkennen kann als den citirten Befunden bei menschlichen Missbildungen, so geschieht es 1) wegen der Kürze der Beobachtungszeit (es handelt sich nur um wenige Tage), welche den Einwand zulässt, dass später doch vielleicht die Muskeln degenerirt wären, und 2) desshalb, weil immerhin kein vollständiger Mangel des Rückenmarks, sondern nur eine Atrophie vorlag, Schaper sagt nur, dass $\gg$ die Nervenzellen, welche im normalen Mark bereits deutlich charakterisirt sind, hier kaum als solche nachweisbar waren « und dass sich "nur in der dorsalen Region hier und da einige große typische Nervenzellen vorfanden ".

Übrigens kann man, glaube ich, in diesen Dingen nicht skeptisch genug sein und so verdient wohl anch ein Bedenken Berücksichtigung, welches jenen erwähnten Fällen von vollständigem Fehlen des Rückenmarks mitsammt seinen motorischen Zellen möglicher Weise entgegengestellt werden könnte, nämlich das, ob hier nicht vielleicht früher vorhandene Rückenmarksreste erst kurz vor der Geburt durch die Maceration in dem bespülenden Fruchtwasser zerstört worden waren? 
Es liegt diese Vermnthung vielleicht um so näher, als thatsächlich in vielen Fällen von Amyelie, wie bekannt, eine nicht zum Rohr geschlossene offene Medullarplatte (Area medullo-vasculosa v. ReckLINGHAUSEA's) persistirt und in derselben unter einem bedeckenden Epithel nicht nur Nervenfasern und Neuroglia; sondern auch Ganglienzellen oder wenigstens diesen nach Größe und Form sehr ähnliche Zellen nachgewiesen werden können. Diesem Einwande gegenüber ist jedoch darauf hinzuweisen, dass, so zart und wenig widerstandsfähig auch diese offen zu Tage liegende Rückenmarksschicht erscheinen mag, doch ihr Bestand unzweifelhaft gesichert ist, so lange sie in vitalem Zustande sich befindet und ihre Ernährung fortdauert, dass also nur im Falle des intrauterinen Absterbens der Frucht eine macerirende und zerstörende Wirkung anf sie von ihrer Umgebung ausgeübt werden kann. In den von mir citirten Fällen ist nun freilich eine ausdrückliche Angabe darüber, ob der Tod schon vor der Geburt eingetreten, nicht gemacht worden; es ist jedoch mit größter Wahrscheinlichkeit anzunehmen, dass dies nicht der Fall gewesen, da anderenfalls die Objekte sicher als ungeeignet zur genaueren mikroskopischen Untersuchung betrachtet worden wären oder wenigstens denselben kein solches Gewicht beigelegt wäre, als es ron Seiten der Untersucher geschehen. Aus demselben Grunde glaube ich auch eine in Folge schlechter Konservirung post partum erfolgte Alteration ausschließen zu dürfen.

Ich betrachte es hiernach also als feststehende Thatsache, dass auch bei wirklicher Amyelie, d. h. nach dem Untergange der sogen. "trophischen Centren « im Rückenmark während der Embryonalperiode die Ernährung und das Wachsthum der quergestreiften Muskeln in normaler. Weise von statten gehen kann; weitere Beweise dafür werden sich in Zukunft wahrscheinlich ergeben, wenn es gelingen sollte, in Fällen von totaler oder partieller Rhachischisis mit persistirender Medullarplatte genau zu ermitteln, in wie weit in derselben der Apparat der motorischen Ganglienzellen in funktionsfähigem Zustande zurïckgeblieben ist, so dass er im Stande ist, einen trophischen Einfluss auf die Muskeln auszuüben. Nach den bisher darüber vorliegenden Angaben scheint mir wenigstens die Vermuthung berechtigt zu sein, dass auch in diesen Fällen meistens trotz vollständig entwickelter Muskulatur ein Deficit in Bezug auf die "trophischen Centren « besteht, eine sichere Entscheidung darüber stößt allerdings vorläufig auf große Schwierigkeiten. 
Alles in Allem ergiebt sich ans dem Gesagten in Betreff der Abhängigkeit der quergestreiften Muskeln von dem centralen Nervensystem das ïberraschende, anscheinend paradoxe Resultat, dass das Verhältnis zwischen beiden im Verlaufe der individuellen Entwickelung in folgender Weise wechselt:

1) Die erste Entwickelung der Muskeln erfolgt unter dem Einfluss der Nervencentra und unter Vermittelung der aus denselben hervorwachsenden motorischen Nervenbahnen (E. H. Weber), eine Selbstdifferenzirung der Maskeln findet nicht statt.

2) Nachdem die Muskeln entstanden, geschieht ihre Ernährung und ihr weiteres Wachsthum während der Embryonalperiode unabhängig von dem Centralorgan, sie haben sich von dem Einfluss desselben emancipirt (LEONOWA, Fraser, Petrín).

3) Erst im postembryonalen Leben stellt sich wieder ein Abhängigkeitsverhältnis her, die »trophischen Centren« des Rückenmarks (und Gehirns) treten in Wirksamkeit.

Ich begnüge mich damit, diesen merkwürdigen Thatbestand zu konstatiren und unterlasse jeden Erklärnngsversuch, der doch nur auf eine Hypothese hinauslaufen könnte.

Dagegen sei nunmehr die weitere Frage, ob etwa ähnliche Verhältnisse auch für die peripherischen motorischen Nerven gelten, Gegenstand der Prüfung. Es ist selbstverständlich, dass wir bei der Entscheidung darüber, ob während der Embryonalzeit ebenso wie im späteren extrauterinen Leben die Nerven unter dem "trophischen " Einfluss der Centralorgane stehen, im Wesentlichen auf dasselbe Beobachtungsmaterial angewiesen sind, welches den vorangegangenen Erörterungen zur Grundlage gedient hat. Leider zeigt dasselbe aber gerade in dieser Beziehung nicht nur vielfache Lücken, sondern auch Widerspriiche, so dass es viel schwieriger ist, sich ein sicheres Urtheil zu bilden, als in Betreff der Muskeln. Einerseits liegen zahlreiche Angaben vor, nach denen trotz mehr oder weniger ausgebildeter Amyelie das periphere Nervensystem in allen Theilen wohlausgebildet war, andererseits ist ein Fehlen der motorischen Bahnen bei diesen Missbildungen beschrieben worden.

Auch hier sei an erster Stelle der sehon erwähnten Beobachtung. E. H. Weber's bei einer Frucht, welcher angeblich » Gehirn und Rịckenmark gänzlich fehlten", gedacht; ohne eine Unterscheidung zwischen vorderen und hinteren Wurzeln zu machen, giebt Weber 
kurzweg an, dass sich »die Wurzeln der Gehirn- und Rückenmarksnerven darstellen ließen und dass die Wurzeln beider Seiten in der Mittellinie aicht zusammenhingen «, ein Fehlen der vorderen Wurzeln bestand also jedenfalls nicht, ein Beobachter, wie WEBER, hätte das sofort bemerkt und notirt; ebenso waren die peripherischen Nerven wohl ansgebildet, wofür einige Maße als Beleg dienen, bei dem nur 11 Par. Zoll langen Embryo war der Stamm des Nervus cruralis $3 \mathrm{~mm}$ breit, $2 \mathrm{~mm}$ dick, Durchmesser des $\mathrm{N}$. phrenicus $1 \mathrm{~mm}$, des $N$. vagus $2 \mathrm{~mm}$.

Eine mikroskopische Untersuchung der Nerven scheint in einem ähnlichen Falle zuerst v. $\mathrm{WAHL}^{1}$ ) ausgeführt zu haben, die Spinalnervenwurzeln waren vorhanden und sollen ebenso, wie die Fasern des Ischiadicus und Vagus ohne Markscheide, aus einer molekularen Masse bestehend sich gezeigt haben.

Mit dieser letzteren Angabe stimmen die Beobachtungen von MaNz (l. c.) keineswegs überein; in seinem interessanten Aufsatze $»$ Das Auge der hirnlosen Missgeburten « sagt er pag. 334: Bei denjenigen Exemplaren, bei welchen auch das Rückenmark fehlte, zeigten nicht nur die N. spinales, sondern auch die Wurzeln markhaltige Fasern, die motorische Wurzel erschien mehr weiß als die sensible, enthielt mehr dunkelrandige Nervenfasern, das Mark erschien auch glänzender als in den sensiblen. Wenn diese sehr bestimmten Angaben tuber die Existenz und gute Entwickelung der vorderen Wurzeln neuerdings von C. und G. Putrón (l. c. pag. 463) in Zweifel gezogen worden sind - es heißt daselbst »eine genauere Beschreibung des Verhaltens der Wurzeln, die eine kritische Prüfung ermöglichte, giebt er (MaNz) nicht, wir müssen daher, um die Möglichkeit des Vorkommens von vorderen Wurzeln bei Amyelie annehmen zu können, noch weitere Beobachtungen abwarten«(!) - so fehlt dazu jede Berechtigung.

Die ersten mikroskopischen Untersuchungen des Rückenmarks bei vollständiger Rhachischisis posterior scheint W. Kocr ${ }^{2}$ ) in mehreren Fällen vorgenommen zu haben. In einem derselben s strahlten von dem aus lockerem, zerfaserten Pia-Duragewebe bestehenden Achsenorgan dicke Nervenbündel gegen das Schwanzende des Fötus aus, sie nehmen ihren Ursprung nur zum kleinsten Theile im Achsenorgan, heben

1) v. WAHL, De retinae textura in monstro anencephalico. Disqu. microsc. Dorpat 1859 . (citirt nach Manz, 1. c.).

2) W. Kocm, Mittheilungen iber Fragen der wissenschaftlichen Medicin.

I. Beiträge zur Lehre von der Spina bifida. pag. 23. 1881. 
in der Mebrzahl frei im Duragewebe an - eine mikroskopische Durchmusterung der aus markhaltigen Fasern bestehenden Nervenstämme und der denselben ansitzenden Ganglien ergiebt keine irgend wie nennenswerthe Abweichung der Norm, in der Medullarplatte selbst aber fehlen zunächst die Ganglienzellen, ebenso entbehrte die mittlere; einem halbgeöffneten Centralkanal entsprechende Furche einer epithelartigen Bekleidung " (welche Косн in einem anderen Falle wohl erhalten gefunden hatte!).

Aus neuerer Zeit liegt ferner der schon erwähnte Fall von Fraser vor, der mir leider im Original nicht zugänglich ist; nach dem SchaPER'schen Citat (l. c. pag. 186) heißt es jedoch daselbst, *A peripherical nervous system was found in all the specimens with well-developed ganglia on the posterior roots « und gleichzeitig wurde konstatirt sthe absence of the ventral cells in the central nervous system ", hiernach würden also trotz fehlenden Riickenmarks Wurzeln und motorische peripherische Nerven bestanden haben.

Ich selbst kann aus eigener Erfahrung nur bestätigen, dass bei der sogen. Amyelie gut ausgebildete vordere Wurzeln vorkommen und dass auch die peripherisehen Nerven dnrehaus keinen Ausfall von Fasern darzubieten brauchen. In einem von mir vor ca. 17 Jahren untersuchten Falle, welcher mir Anlass zu dem oben erwähnten Vortrage gab, war der Befund folgender:

"Das Schädeldach fehlte vollstïndig, das Gehirn war, was sonst gewöhnlich nicht der Fall ist, ziemlich gut ausgebildet, wenigstens ließ das an Größe zurïckgebliebene Großhirn deutlich eine Abtheilung in zwei Hemisphären und innerhalb derselben die Seitenventrikel erkennen, die basalen Theile des Gehirns schienen dagegen gänzlich zu fehlen. An der Wirbelsänle fehlen sämmtliche Wirbelbogen and die Wirbelkörper liegen, eine weit offene Rinne bildend, zu Tage. Diese Rinne ist ausgekleidet von den Riickenmarkshüuten und zwar befand sich die Dura mater in ziemlich fester Verbindung mit den Knochen und ging an den Seitentheilen in das Periost derselben über, die weichen Häute liegen nur ganz locker der Dura mater anf und gehen an den Seiten in die äußeren Hautbedeckungen über; sucht man sie abzulösen, so spannen sich jederseits zwei Reihen von Nervenwurzeln aus, welche von der «̈ußeren Seite der Häute zu den Foramina intervertebralia verlaufen, eine äußere Reihe den hinteren Wurzeln entsprechend, eine innere Reihe die vorderen Wurzeln darstellend. Die freie, wach oben gekehrte Oberfläche der Häute hat in der Mitte da, wo das anscheinend ganz fehlende Rückenmark ihr anfliegen sollte, ein geröthetes schleimhautähnliches Aussehen und es lässt sich konstatiren, dass hier eine dünne Schicht röthlicher breiiger Substanz den Hänten anfliegt, übrigens war vom Rückenmark nichts zu sehen mit Ausnahme des Filum terminale, welches inmitten der Cauda equina verläuft. Die Wurzeln, vor dere sowohl als hintere, haben ein gutes Anssehen, sind krätig entwiekelt, 
sie gehen in gewöhnlicher Weise in die normal beschaffenen Spinalganglien über und auch in den peripheren Theilen, so weit ich sie untersucht habe, zeigen sich die Spinalnerven so beschaffen, wie man sie sonst zu finden pflegt.

Die mikroskopische Untersuchng bestätigte denn auch, dass die spinalen Nerven (ich habe eine große Zahl derselben sowohl frisch als mit Osinium untersucht) vollständig normale Fas e rn enthielten, nirgends fand ich das bekannte Bild der Nervendegeneration, wie sie etwa Durchschneidungen der Nerven folgt; dasselbe gilt auch für die Nervenwurzeln, die sich in die Rïckenmarkshänte inseriren, und zwar sowohl für vordere als auch hintere Wurzeln. Die Untersuchung der Rückenmarkshäute ferner und der ihnen aufgelagerten Masse ergiebt, dass zwar von einem vollständigen Mangel des Rückenmarks nicht die Rede sein kann, jedenfalls war dasselbe aber äußerst rudimentär ausgebildet, es stellt sich nümlich als eine über den Rückenmarkshäuten ausgebreitete, an verschiedenen Stellen verschieden dicke, in maximo aber immer nur einen Bruchtheil eines Millimeters starke Schicht dar, welche aus einer körnigfaserigen neurogliaartigen Masse besteht, diese ist, ebenso wie die Rückenmarkshänte, von zahlreichen weiten Gefäßen durchsetzt, zeigt auch vielfach Blntungen in ihre Substanz und ist interessanter Weise an ihrer freien Oberfläche meistens dentlich bedeckt von einer zarten, kurzcylindrischen Epithelschicht, offenbar dem Epithel des Centralkauals des Ruickenmarks entsprechend. Fon Elementen ansgesprochen nervöser Natur fand ich in dieser Substanz nur wenig, aber allerdings waren hier und da markhaltige Nervenfasern und auch, wie es schien, ziemlich spälliche große Zellen mit rundem Kern und Kernkörperchen, die den Habitus von Ganglienzellen hatten, vorhauden, sie waren jedoch abgerundet und ließen keine Fortsäitze erkennen.«

Eine zweite, ganz ähnlich gestaltete Missbildung habe ich etwas später zu untersuchen Gelegenheit gehabt und mich davon überzeugen können, dass hier das Riickenmark und die aus ihm entspringenden Nerven einen vollständig gleichen Befund darboten; der gangliöse Charakter der in der Medullarplatte sich vorfindenden Zellen war in Folge ihrer sehr bedeutenden Größe (bis $0,066 \mathrm{~mm}$ Durchmesser) und deutlich ausgebildeten Fortsätze noch mehr ausgeprägt. Ich bemerke ausdriicklich, dass beide Frïhte in ganz frischem Zustande in meine Hände gelangten, so dass sich die makroskopischen sowohl als die mikroskopischen Untersuchungsresultate mit aller wünschenswerthen Sicherheit erheben ließen. Durchaus berechtigt scheint es mir demnach, wenn in den Lehrbiichern iiber Missbildungen (Foerster, AmLfeld) angegeben wird, dass bei den Zuständen der Anencephalie und Amyelie Hirn- und Rückenmarksnerven vollständig vorhanden zu sein pflegen, und es beruht jedenfalls auf einem Irrthum, wenn nenerdings MoNarow ${ }^{1}$ ) sich dahin

1) Monakow, Missbildungen des Centralnervensystems, in Ergebnissen der allgem. Pathologie und pathol. Anatomie, herausgeg. von LUBARsCH und OsTERTAG. Bericht f. 1899 (erschienen im Juli 1901). pag. 523. 
geäußert hat, dass in denjenigen Fällen von Amyelie, in welchen „vom Medullarrohr die ventralen Abschnitte zwar zur duirftigen Entwickelung gelangt sind, die Medullarplatte aber nicht zum Medullarrohr sich geschlossen hat", die vorderen Wurzeln nur mikroskopisch noch nachweisbar seien und wenn er weiterhin sogar von einem sausnahmslosen Fehlen der vorderen Wurzeln bei Amyelie spricht, wodurch die Richtigkeit der Lehre, dass sie aus dem Medullarrohr hervorwachsen, bestätigt würde.

Dass auch bei partiell mangelhafter Ausbildung des Rückenmarks die betreffenden Nervenwurzeln und ihre peripherisehen Fortsetzungen häufig gut entwickelt getroffen werden, lehren ferner die Beobachtungen ïber Spina bifida. So spärlich allerdings auch hier genau mikroskopisch untersuchte Fälle immer noch sind, so wird es doch genïgen, zum Beweise hierfür auf folgende, der neueren Litteratur entnommene Beispiele hinzuweisen.

v. Recklinghausen ${ }^{1)}$ fand in dem von ihm beschriebenen Fall II (pag. 302) bei partieller Rhachischisis in der Area medullo-vasculosa nur spärliche, theils vereinzelte, theils gruppirte Ganglienzellen und myelinhaltige zerstreute oder bündelförmige Nervenfasern, giebt aber an, dass vordere und hintere Nervenwurzeln gut entwickelt waren, wie auch seine Abbildung Taf. X Fig. 5 erkennen lässt.

Ebendaselbst bot in Fall IV der Fötus gleichfalls eine Rhachischisis dorsalis dar, trotzdem entspringen aus der Area medullovasculosa vier Reihen kräftiger Nervenwurzeln (pag. 308).

Dagegen zeigte sich in den Fiallen X, XI und XII, in denen eine Myelomeningocele mit starker Flüssigkeitsansammlung vorlag, allerdings, dass $\gg$ die vorderen Nervenwurzeln großentheils abhanden gekommen waren ", doch scheint $v$. Rechlingriausen geneigt, dies auf eine »atrophirende Degeneration" zu beziehen, welche nicht aus der rudimentären Entwickelnng des Rückenmarks, sondern vielmehr aus der Emporzerrung und Dehnung, welche die Nerven durch die Fluissigkeitsansammlung erfahren hatten, hervorgegangen war, er sagt, »zu den sekundären Metamorphosen ist auch die Atrophie der Nervenwurzeln zu rechnen, die im Allgemeinen mit der Dehnung, die sie erfahren, gleichen Schritt halten diurfte« (pag. 329, 447).

Aus der Arbeit von C. und G. Pexrén (l. c.) sei ferner besonders Fall IV (pag. 364) hervorgehoben, es bestand hier Anencephalie

1) v. Reckutinghasen, Untersuchungen über die Spina bifida. Vircnow's Arehiv. Bd. 105. 1886. 
mit partieller Rhachischisis cervicalis, der Centralkanal öffnete sich oben und der mediale Theil der hinteren Fläche des Rüekenmarks erhielt eine epitheliale Bekleidung, hier lassen sich zunächst noch Vorderhörner mit körnigen, stark zerfallenen Resten von Ganglienzellen erkennen, vordere und hintere Wurzeln erscheinen "gut entwickelt ; noch weiter aufwärts wird das Rückenmark ganz rudimentär und fehlt schließlich, »den einzigen Rest bildete bier und da an der hinteren Fläche vorhandenes Epithel «, »im obersten Theil des Wirbelkanals besteht also wahre Amyelie « trotzdem treten auch hier "gut entwickelte Wurzeln * hervor ${ }^{1}$ ).

Bemerkenswerth ist auch die ron $C$. und G. Pexrén bei der Beschreibung ihres Falles I (pag. 347), wo es sich ebenfalls um eine mit Rhachischisis cervicalis kombinirte Anencephalie handelte, gemachte Angabe, dass an Schnitten, welche durch die vorderen Theile der auf der Basis cranii gelagerten rothen Masse gefiihrt wurden, "keine Ganglienzellen mehr zu finden waren, leidlich gut entwickelte Wurzeln aber immer noch existirten $\ll$.

Andererseits sind nun aber allerdings auch, wie sehon oben gesagt, von einzelnen Autoren sehr bestimmte Angaben dariiber gemacht worden, dass Missbildungen des Rückenmarks mit einem entsprechenden Defekt des peripherischen Nervensystems, speciell der motorischen Bahnen, einhergehen; auf diese Fälle hat sich Monakow am angeführten Orte gestützt und ist, dieselben einseitig beriicksichtigend, zn einer irrthümlichen Auffassung gelangt. Hierher würden gehören die oben eitirten Fälle von Leonowa und den Brüdern Petrén.

Wie schon gesagt, fehlten in der LEovowA'schen Beobachtung Gehirn und Rückenmark vollständig, in der Cervicalgegend und in der Nähe des »rerwachsenen" (!?) Foramen occipitale magnum befand sich ein rothes, schwammiges Gewebe, »der Wirbelkanal ist in seiner ganzen Ausdehnung ausgefuillt von Nervenwurzehn, namentlich von denen der Canda equina, einzelne Wurzeln durchsetzen ununterbrochen den ganzen Wirbelkanal und eine den caudalsten Ganglien entstammende Wurzel legt sich schließlich auf den Occipitalknochenk, » es liegen hier wohl die für den intramedullären Verlauf bestimmten Abschnitte isolirt und frei (?), »die vorderen Wurzeln fehlen vollständig", "vergleicht man auf derselben Höhe angebrachte

1) Diese von den Herren PexRÉn gemachte Beobachtung lü̈tte sie wohl darauf hinweisen sollen, dass ihre oben erwähnten Zweifel an der Richtigkeit der damit durchaus ïbereinstimmenden MaNz'schen Angaben (s. o.) nicht stichhaltig* sind. 
Querschnitte durch den Nervus ischiadicus bei unserem Präparat mit solchen von einem (gleich großen) normalen Fötus, so erscheinen die Dimensionen des letzteren wesentlich größer (Durchmesser dort 8 resp 16 , hier 10 resp. 18), diese Differenz wird begreiflich, wenn man berïcksichtigt, dass bei dem pathologischen Präparat der Antheil der motorischen Fasern fehlter.

In Bezug anf die Deutung, welche Leonowa dieser Beobachtung gegeben hat, lassen sich, wie mir scheint, gewisse Bedenken nicht unterdrücken. Wenn auch nicht bezweifelt werden kann, dass hier die vorderen Nervenwurzeln nicht in normaler Weise vollzählig ausgebildet waren, so diurfte es doch fraglich sein, ob dieselben vollständig fehlten, anffällig ist die reich ausgebildete Cauda equina, deren Zusammensetzung aus einer verminderten Zahl von Nerven nach Beschreibung and Abbildung kaum wahrscheinlich ist; befremdend ist auch die Annahme, dass ein Theil der den Wirbelkanal erfüllenden Nervenstränge aus den isolirten intramedullären Abschnitten der Riickenmarksfasern bestehen soll, da ein solcher Befund bei einer mit Anencephalie verbundenen Amyelie sonst, so weit mir bekannt, in keinem Falle erwähnt worden ist. Dagegen aber, dass in den peripheren Nerven der motorische Antheil von Fasern ganz ausgefallen war, lässt sich anführen, dass diese Nerven doch eine sehr ansehnliche Stärke besaßen und die Differenz gegenüber den Nerven normaler Föten als ziemlich unbeträchtlich bezeichnet werden darf, keineswegs anf die Hälfte oder ein Dritttheil, wie man hiernach erwarten sollte, heruntergegangen war. Ich kaun daher jedenfalls SCHAPER (1. c. pag. 187) nicht beistimmen, wenn er behauptet, dass "Leovowa durch die mikroskopiscbe Untersuchung den positiven Beweis erbracht habe, dass die Zahl der Fasern in den peripheren Nerven in der That herabgesetzt war und dass dieses Deficit auf Rechnung der fehlenden vorderen Wurzeibiindel zu setzen sei«, und ebensowenig kann ich ihm darin folgen, wenn er aus der Beobachtung den Sehluss ableitet, dass die peripheren Nerven »ausschließlich aus den peripheren Fortsätzen der sensorischen cerebralen ụnd spinalen Ganglienzellen zusammengesetzt gewesen seien «.

Der Petrén'sche Fall von Anencephalie und totaler Amyelie, in welchem ebenfalls anch mikroskopisch keine Spur von Rückenmark entdeckt werden konnte, ist nur kurz beschrieben, es wird angegeben, dass von den Spinalganglien $\gg$ Nerven ihren Ursprung nahmen theils in der Richtung nach vorn, welche man durch die Intervertebrallöcher verfolgen kann, theils nach hinten, die letzteren gehen 
durch die Dura, alsdann theilt sich jede Nervenwurzel in einige wenige Stränge von 0,5 bis $1 \mathrm{~mm}$ Durchmesser, sie können im Allgemeinen $2-3 \mathrm{~cm}$ weit verfolgt werden, verlieren sich danach aber in einer sie überlagernden dünnen Membran «-..- - jede Bildung, welche als vordere Wurzeln gedentet werden könnte, wird vermisst - über die peripherischen Nerven wird nichts berichtet.

Als dritter Fall, in welchem nach einem Citat in der Petrénschen Arbeit (pag. 463) ebenfalls mit dem Rückenmark gleichzeitig die vorderen Wurzeln gefehlt haben sollen, wäre ferner der von GADE anzufuhren, die Originalabhandlung habe ich mir jedoch nicht verschaffen können.

Überblicken wir sämmtliche hier zusammengestellten Beobachtungen, so muss zugestanden werden, dass sie nicht genügen, um vollständige Klarheit über die Frage, in wie weit im embryonalen Leben die peripherischen motorischen Nerven von den Centren abhüngig sind, zu versehaffen. Dürfen wir es anch durch die letztgenannten Mitheilungen von Leonowa, Perrén und Gade als festgestellt ansehen, dass sich mit dem durch Bildungsfehler bedingten vollständigen Untergange des Rückenmarks ein Fehlen motorischer Wurzeln verbinden kann, so ist durch dieselben doch nicht ein strikter Beweis dafür geliefert, dass letztere in Folge der Zerstörung der centralen motorischen Ganglienzellen durch degenerative Atrophie zu Grunde gegangen sind, während die den Spinalganglien entstammenden hinteren Wurzeln zu normaler Ausbildung gelangten; ganz abgeseben davon nämlich, dass nach unseren Erfahrungen über degenerative Atrophie im extrauterinen Leben dieselbe nie zn einem vollständigen Schwunde, sondern selbst in den extremsten Fällen nur zu einer Umwandlung derselben in Bindegewebsstränge fithrt, ist die Möglichkeit nicht ausgeschlossen, dass dieselbe Störung; welehe das Riickenmark in jenen Fällen betroffen hatte, auch auf die motorischen Wurzeln eingewirkt und ihren Untergang herbeigeführt hat, einer solchen Annahme steht die gute Exhaltung der hinteren Wurzeln keineswegs entgegen, da die Entwickelungsgeschichte lehrt, dass letztere später sich entwickeln als die vorderen Wurzeln (Hertwig, Lebrb. d. Entwickelungsgeschichte. 5. Aufl. pag. 435), jene pathologische Störung also in einen Zeitranm gefallen sein kann, wo sie noch nicht existirten, da ferner aber auch die hinteren Wurzeln, falls sie ebenfalls zerstört worden, sich von den erhaltenen Spinal. ganglien aus wieder regenerirt haben konnten. Was aber das Verhalten der peripherischen motorischen Bahnen betrifft, so entbehrt, 
Über d. Beziehungen d. Nerven u. Muskeln zu d. Centralorganen beim Embryo. 471

wie aus dem oben Gesagten hervorgeht, in dem LEonowa'schen Falle - dem einzigen, welcher in dieser Beziehung in Betracht kommt, da er allein Angaben über das periphere Nervensystem enthält die Behauptung, dass hier sämmtliche motorische Bahnen in Folge des Verlustes ihrer trophischen Centren ausgefallen wären, einer hinreichenden Begriundung.

Auf der anderen Seite miissen wir nun aber mit der durch zahlreiche Beobachtungen erhärteten Thatsache rechnen, dass bei den hochgradigsten Bildungsfehlern des Rückenmarks der ganze Apparat der motorischen Nervenwurzeln und zugleich wahrscheinlich auch der peripherischen motorischen Nerven voll entwickelt ist, dass Zeichen einer jeden degenerativen Atrophie in ihm fehlen können. In wie weit in diesen Fällen in den Rückenmarksresten die ventralen motorischen Ganglienzellen erhalten geblieben waren, lässt sich schwer beurtheilen, meistens schienen dieselben jedoch nur spärlich vorhanden zu sein, so dass ihre Zahl kaum genügte, um sämmtliche periphere Leitungsbahnen zu innerviren, in einigen Fällen (W. Kocn, Fraser, Fall IV von Petrén) ist sogar ausdruicklich angegeben, dass jene Zellen ganz fehlten. Wir werden demnach den Gedanken nicht zurückweisen können, dass hier andere Gesetze gelten als für das spätere extrauterine Leben, und kommen zu dem Resultate,

dass wahrscheinlich bei dem Embryo die peripherischen motorischen Nerven, ebenso wie es für die quergestreiften Muskeln gilt, zu ihrer Ernährung und ihrem Wachsthum des Einflusses der strophischen Centren im Rückenmark (und Gehirn) nicht bedürfen.

So auffällig dieses Ergebnis ist und so wenig einstweilen ein Einblick in die physiologischen Vorgänge, welche uns dasselbe erklären könnten, gestattet ist, so steht es doch nicht ganz ohne Analogie da, es sei an die bekannte Thatsache erinnert, dass auch im Zustande des Winterschlafs der Thiere der Einfluss der trophischen Centren auf die Ernährung der peripherischen Nerren suspendirt zu sein scheint, Durchschneidungen von Nerven bei Winterfröschen haben keine Degeneration des abgetrennten peripherischen Theils zur Folge oder es tritt eine solehe wenigstens erst sehr spät auf und bleibt auf sehr geringfügige Veränderungen beschränkt, auch bei winterschlafenden Säugethieren (Ziesel) habe ich ähnliche Erfahrungen gemacht. Ein befriedigendes Verständnis der für den Embryo geltenden Verhältnisse eröffnet sich freilich damit noch nicht, dass wir dieselben mit denen winterschlafender Thiere parallelisiren. 
472 E. Neumann, Über die Beziehungen der Nerven und Muskeln etc.

Vielleicht geben diese Betrachtungen aber anch dazu Veranlassung, die Lehre von den strophischen Centren«, so allgemein dieselbe auch anerkannt ist, nochmals einer eingehenden Revision zu unterziehen; dass auch gewisse andere pathologische Erfahrungen mit ihr nur schwer in Einklang zu bringen sind, diurfte nicht zu leugnen sein, ich weise z. B. auf eine von CoHnHerm (Allgem. Path. I. pag. 599) angeführte Beobachtung ron ERB und Schultze hin, wo sich bei spinaler Paralyse hochgradige Atrophie der Maskeln und degenerative Veränderungen der Ganglienzellen im Ruckenmark ohne nachweisbare Veränderungen der peripheren Nerven und der vorderen Wurzeln vorfanden.

Rauschen, den 22. August 1901. 